\title{
Dempster-Shafer's Evidence Theory-based Edge Detection
}

\author{
Suk Tae SEO ${ }^{1}$, Krishnamoorthy SIVAKUMAR ${ }^{2}$ and Soon Hak KWON ${ }^{*}$ \\ ${ }^{1}$ Department of Electrical Engineering, Yeungnam University, Republic of Korea \\ ${ }^{2}$ School of EECS, Washington State University, Pullman WA, USA \\ *Tel:+82-53-810-3514, Fax:+82-53-810-4767, E-mail:shkwon@yu.ac.kr
}

\begin{abstract}
Edges represent significant boundary information between objects or classes. Various methods, which are based on differential operation, such as Sobel, Prewitt, Roberts, Canny, and etc. have been proposed and widely used. The methods are based on a linear convolution of mask with pre-assigned coefficients. In this paper, we propose an edge detection method based on Dempster-Shafer's evidence theory to evaluate edgeness of the given pixel. The effectiveness of the proposed method is shown through experimental results on several test images and compared with conventional methods.
\end{abstract}

Key words : Edge detection, Evidence theory, Dempster-Shafer

\section{Introduction}

Edge is a significant element to represent boundary of objects from given image. Moreover edge could be applied to segmentation problem, shape analysis, and other image processing problems, because edge contains various characteristic information of objects in image. That is, edge is used as important information to analyze image.

Various edge detection methods based on differential operation have been proposed and widely used such as Sobel, Prewitt, Canny, Laplacian, and etc [1]-[4]. These conventional methods detect edge based on linear convolution mask with pre-assigned coefficients [5]. Therefore, the performance of edge detection depends on the type of mask and coefficients. Furthermore, some conventional methods detect double edges in the case of one-pixel-width edges, due to the type of mask used [5]. Thus, to overcome these drawbacks, an edge detection method is needed that is not dependant on a fixed mask with coefficients, but rather based on the information obtained data of the given image.

Based on the needs, edge detection methods have been proposed using Dempster-Shafer's theory [7], [8]. DempsterShafer's theory is an evidence theory to reduce the uncertainty by combining the evidences obtained from different sources [6]-[11]. Therefore the theory is an appropriate method to deal with uncertainties of image such as edge, noise, and etc. However these methods [7], [8] have the characteristics of the conventional edge detection operations, because the methods are based on Sobel edge operation. Thus, in this paper, we propose an edge detection method which is based on the possibility of edgeness based on the Dempster-Shafer's theory. Using the Dempster-Shafer's theory, the possibility for a pixel is determined by combining the possibilities of edgeness

Manuscript received Nov. 6, 2010; revised Mar. 7, 2011;

Accepted Mar. 12, 2011.

* Corresponding author obtained from the different hypotheses.

To show the effectiveness of the proposed method, the edge detection experimental results on several images and the comparison with the typical conventional edge detection methods(Sobel, Prewitt, and Canny) are provided. From the experimental results, it is shown that the proposed method is similar or better than the conventional methods.

\section{Preliminary}

\subsection{Conventional edge detection methods}

Edge is a boundaries of objects which often lead to change of intensity in image. Typical edge in image processing could be classified into 4 types as follows [1].

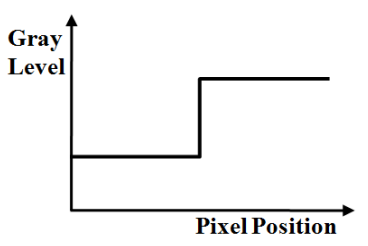

(a)Step edge

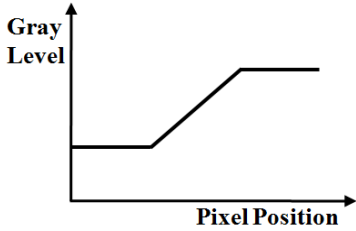

(c)Ramp edge

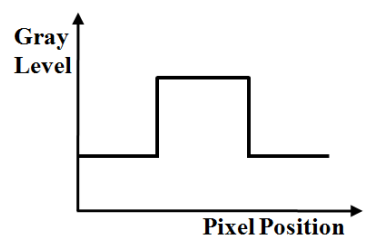

(b)Line edge

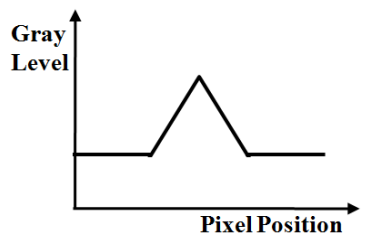

(d)Roof edge
Figure 1. Four types of edge

Various conventional methods, which are using the difference of intensity, have been proposed such as Sobel, Prewitt, and Canny. Edge detection operations of Sobel and Prewitt are summarized in Table 1. 
Table 1. Edge detection operations of Sobel and Prewitt

\begin{tabular}{|c|c|c|c|c|c|c|}
\hline & \multicolumn{3}{|c|}{ Horizontal } & \multicolumn{3}{|c|}{ Vertical } \\
\hline \multirow{3}{*}{ Sobel } & -1 & -2 & -1 & -1 & 0 & 1 \\
\hline & 0 & 0 & 0 & -2 & 0 & 2 \\
\hline & 1 & 2 & 1 & -1 & 0 & 1 \\
\hline \multirow{3}{*}{ Prewitt } & -1 & -1 & -1 & -1 & 0 & 1 \\
\hline & 0 & 0 & 0 & -1 & 0 & 1 \\
\hline & 1 & 1 & 1 & -1 & 0 & 1 \\
\hline
\end{tabular}

Edge is detected by convolution between image and operations shown in Table 1. If the coordinate of the subject pixel in image is $(x, y)$, then the edgeness of the pixel is evaluated from the Equation (1).

$$
\begin{gathered}
E_{m}(x, y)=\sqrt{\left(G_{H}(x, y)\right)^{2}+\left(G_{V}(x, y)\right)^{2}} \\
\text { Or } \\
E_{m}(x, y)=\left|G_{H}(x, y)\right|+\left|G_{V}(x, y)\right|
\end{gathered}
$$

where $G_{H}$ and $G_{V}$ represent convolution results according to methods shown in Table 2.

Table 2. $G_{H}$ and $G_{V}$ according to methods

\begin{aligned} \hline \hline Sobel \\ $G_{H}(x, y)=f(x+1, y-1)+2 f(x+1, y)+f(x+1, y+1) \\ -f(x-1, y-1)-2 f(x-1, y)-f(x-1, y+1) \\ G_{V}(x, y)=f(x-1, y+1)+2 f(x, y+1)+f(x+1, y+1) \\ -f(x-1, y-1)-2 f(x, y-1)-f(x+1, y+1) \\$\hline Prewitt \\ \hline$G_{H}(x, y)=f(x+1, y-1)+f(x+1, y)+f(x+1, y+1) \\ -f(x-1, y-1)-f(x-1, y)-f(x-1, y+1) \\ G_{V}(x, y)=f(x-1, y+1)+f(x, y+1)+f(x+1, y+1) \\ -f(x-1, y-1)-f(x, y-1)-f(x+1, y+1)\end{aligned}$

In Canny's method [2], it applies firstly two-dimensional Gaussian function to reduce noise from image, and then detect edge using Sobel's operations. The Gaussian function and example are shown in Equation (4) and Figure 2.

$$
G(x, y)=e^{\left(-\frac{x^{2}+y^{2}}{2 \sigma^{2}}\right)}
$$

\begin{tabular}{c|c|c|c|c|c|}
\hline \multicolumn{1}{c|}{$\frac{1}{115}$} & 4 & 5 & 4 & 2 \\
\hline 4 & 9 & 12 & 9 & 4 \\
\hline 5 & 12 & 15 & 12 & 5 \\
\hline 4 & 9 & 12 & 9 & 4 \\
\hline 2 & 4 & 5 & 4 & 2 \\
\hline
\end{tabular}

Figure 2. Example of Gaussian function with $\sigma=1.4$

\subsection{Dempster-Shafer's Evidence Theory}

\subsubsection{Fundamental Theory}

The purpose Dempster-Shafer's evidence theory is to reduce the uncertainty by combining the evidences from the different sources [6], [9]. Let $X$ is the universal set and the power set of $X$ is $2^{X}$. A basic probability assignment(i.e. mass function $m: 2^{X} \rightarrow[0,1]$ ) satisfies Equation (5) [9], [10]:

$$
\left\{\begin{array}{l}
m(\phi)=0 \\
\sum_{A \subseteq X} m(A)=1
\end{array}\right.
$$

where $\phi$ is an empty set.

The value of $m(A)$ is the belief that certain elements of $X$ belongs to the exact set $A$, not to the subset of $A$. That is, $m(A)$ corresponds to the weight of evidence in favor of $A$ exactly and fully [6]-[11]. From the mass function, the interval of upper and lower bounds are defined, which are called belief( $b e l)$ and plausibility ( $p l$ ), as follows:

$$
\begin{aligned}
& \left\{\begin{array}{l}
\operatorname{bel}(\phi)=0 \\
\operatorname{bel}(A)=\sum_{B \subseteq A} m(B)
\end{array}\right. \\
& \left\{\begin{array}{l}
p l(\phi)=0 \\
\operatorname{pl}(A)=\sum_{B \cap A \neq \phi} m(B)
\end{array}\right.
\end{aligned}
$$

where $A, B \subseteq X$. From Equations (6) and (7), the relationship of the two measures are defined as follows:

$$
\operatorname{pl}(A)=1-\operatorname{bel}(\bar{A})
$$

where $\bar{A}$ is the complement set of $A$ and it satisfies that $\bar{A}=X-A$.

\subsubsection{Combination Rule}

Dempster-Shafer's evidence theory also provides combining method of evidences from the different sources. If there are two masses $m_{1}$ and $m_{2}$ obtained from the different sources, then the combined mass $m_{1,2}$ is defined by the orthogonal sum of the two masses $m_{1}$ and $m_{2}, \quad m_{1,2}=m_{1} \oplus m_{2}$. That is, the new mass $m$ which is combined with $n$ number of masses is defined as follows:

$$
\left\{\begin{aligned}
m(\phi) & =0 \\
m(A) & =\left(\left(\left(m_{1} \oplus m_{2}\right) \cdots\right) \oplus m_{n}\right) \\
& =\frac{1}{1-\sum_{A_{1} \cap \cdots \cap A_{n}=\phi} \prod_{i=1}^{n} m_{i}\left(A_{i}\right)} \sum_{A_{1} \cap \cdots \cap A_{n}=A} \prod_{i=1}^{n} m_{i}\left(A_{i}\right)
\end{aligned}\right.
$$

where $1-\sum_{A_{1} \cap \cdots \cap A_{n}=\phi} \prod_{i=1}^{n} m_{i}\left(A_{i}\right)$ is the normalization factor that can eliminate the effect of the masses associated with the null set. 


\section{Proposed Method}

Let $Z=\left\{z_{1}, \cdots, z_{9}\right\}$ be a finite universal set representing grey levels in the $3 \times 3$ local region and $Z_{(\cdot)}=\left\{z_{(1)}, \cdots, z_{(9)}\right\}$ be a permutation set of the elements of $Z$ such that $z_{(1)} \leq \cdots \leq z_{(9)}$. To evaluate edgeness, we use the differences of partitioned regions based on maximum differences between $z_{(i+1)}$ and $z_{(i)}$, $i=1, \cdots, 8$ and possibilities obtained from two different evidences defined in this paper.

\subsection{Region Partition and Difference}

The algorithm to partition $3 \times 3$ local region at the subject pixel $(x, y)$, and to obtain the differences of partitioned regions is f as follows:

Step 1. From the $3 \times 3$ local region at the subject pixel $(x, y)$, find $T=z_{(i)}$ with $\max _{i=1, \cdots, 8}\left\{z_{(i+1)}-z_{(i)}\right\}$ of the local region.

Step 2. Partition the $3 \mathrm{X} 3$ local region into two regions $\left(C_{1} \geq T, C_{2}<T\right)$ with $T$. The example of region partition is shown in Figure 3.

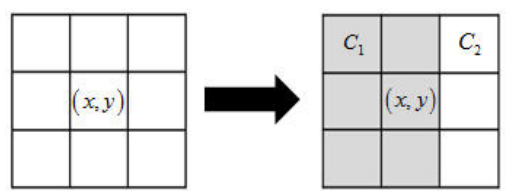

Figure 3. Example of region partition.

Step 3. Calculate the difference by using Equation (10).

$$
\operatorname{dif}(x, y)=\max _{i=1, \cdots, 8}\left\{z_{(i+1)}-z_{(i)}\right\}
$$

\subsection{Possibility of Edgeness}

To apply Dempter-Sherfer's evidence theory, we have defined two elements noise $(N)$ and edge $(E)$. The power set is $\operatorname{noise}(N)$, edge $(E)$, universe $(N \cup E, N E)$, and empty $\operatorname{set}(\phi)$. Moreover, to assign the possibility of edgeness for the subject pixel, we have defined two mass functions as follows:

Definition 1. If we consider two cases, noise and edge, for the subject pixel, then we can define that the possibility to be a noise signal of the subject pixel is decreased as the number of pixels in the class which contains the subject pixel is increased.

Based on Equation (5) and the definition 1, the mass function $m_{1}$ is defined as follows [9]:

$$
\left\{\begin{array}{l}
m_{1}(\phi)=0 \\
m_{1}(N)+m_{1}(E)+m_{1}(N E)=1
\end{array}\right.
$$

$$
\left\{\begin{array}{l}
m_{1}(E)=\frac{\max _{i=1, \cdots, 8}\left\{z_{(i+1)}-z_{(i)}\right\}}{z_{(9)}-z_{(1)}} \\
m_{1}(N)=\alpha\left\{1-m_{1}(E)\right\} \\
m_{1}(N E)=(1-\alpha)\left\{1-m_{1}(E)\right\}
\end{array}\right.
$$

where $I(x, y)$ is the number of pixels in the class which contains the subject pixel, and $\alpha$ is a weight constant between $[0,1]$.

Definition 2. If we consider two cases, noise and edge, for the subject pixel, then we can define that the possibility to be a noise signal of the subject pixel is decreased as the connectivity between the subject pixel and neighbor pixel is increased. Therefore we have evaluated the connectivity, when the combined local regions, as shown in Figure 2, are partitioned with mean value $T^{\prime}$ of combined regions, if the subject pixel $(x, y)$ of local region and the neighbor pixel $\left(x^{\prime}, y^{\prime}\right)$ of the neighbor region are classified into same class, then the subject pixel has connectivity with the subject pixel of the neighbor region. The example is shown in Figure 4 and 5 to evaluate the connectivity.

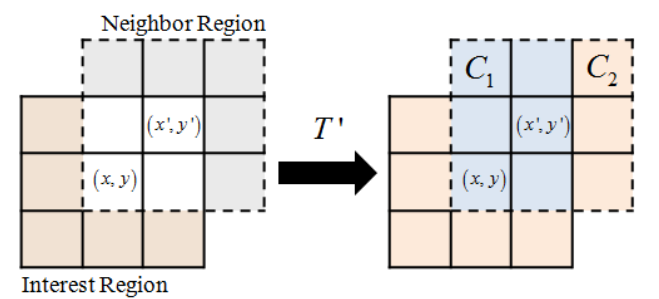

Figure 4. Example when the subject pixel $(x, y)$ have connectivity with neighbor pixel $\left(x^{\prime}, y^{\prime}\right)$

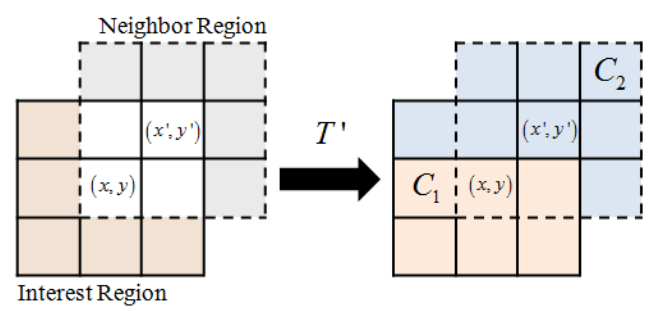

Figure 5. Example when the subject pixel $(x, y)$ does not have connectivity with neighbor pixel $\left(x^{\prime}, y^{\prime}\right)$

From Figure 4 case, if the subject pixel $(x, y)$ and neighbor pixel $\left(x^{\prime}, y^{\prime}\right)$ are classified into same class, then the subject pixel $(x, y)$ has connectivity with neighbor pixel $\left(x^{\prime}, y^{\prime}\right)$. In that case, the connectivity $C(x, y)$ is increased ' +1 '.

Oppositely, from Figure 5, if the subject pixel $(x, y)$ and neighbor pixel $\left(x^{\prime}, y^{\prime}\right)$ are classified into other classes, then the subject pixel $(x, y)$ does not has connectivity with neighbor pixel $\left(x^{\prime}, y^{\prime}\right)$. 
Based on the algorithm, we evaluate connectivity between the subject pixel of interest region and the neighbor pixels of the every neighbor regions. Based on the connectivity, mass function $m_{2}$ is defined as follows:

$$
\begin{aligned}
& \left\{\begin{array}{l}
m_{2}(\phi)=0 \\
m_{2}(N)+m_{2}(E)+m_{2}(N E)=1
\end{array}\right. \\
& \left\{\begin{array}{l}
m_{2}(N)=1-\frac{C(x, y)}{9} \\
m_{2}(E)=\alpha\left\{1-m_{2}(N)\right\} \\
m_{2}(N E)=(1-\alpha)\left\{1-m_{2}(N)\right\}
\end{array}\right.
\end{aligned}
$$

where $C(x, y)$ is the connectivity of subject pixel $(x, y)$, and $\alpha$ is a weight constant between $[0,1]$.

\subsection{Combination Rule and Edge Magnitude}

Possibilities obtained from the definition 1 and 2 are needed to combine to determine the final possibility of edgeness. The combination rules are shown in Table 1.

Table 1. Combination rules

\begin{tabular}{cccccc}
\hline \multirow{3}{*}{$m_{1,2}$} & & \multicolumn{3}{c}{$m_{1}$} \\
\cline { 3 - 5 }$m_{2}$ & & $N$ & $E$ & $N E$ \\
\hline & $N$ & $N$ & $\phi$ & $N$ \\
\cline { 2 - 5 } & $E$ & $\phi$ & $E$ & $E$ \\
\cline { 2 - 5 } & & $N E$ & $N$ & $E$ & $N E$ \\
\hline
\end{tabular}

From the combination rules in Table 1, we extract the case of ' $E$ ', and the combined possibility of edgeness for the subject pixel is defined as follows:

$$
P_{E}(x, y)=\frac{\sum_{A \cap B \in E} m_{1}(A) m_{2}(B)}{1-\sum_{A \cap B=\phi} m_{1}(A) m_{2}(B)}
$$

Based on the possibility obtained from Equation (15) and the difference from Equation (10), we determine the final edge magnitude by using Equation (16).

$$
M_{E}(x, y)=\operatorname{dif}(x, y) P_{E}(x, y)
$$

\subsection{Threshold Value Selection}

To obtain edge image from the edge magnitude image, thresholding is necessary. Therefore, in this letter, we select the threshold value based on the center of gravity. To determine the threshold value, the edge magnitude values are expanded into range $[0,255]$ with a linear stretch function, and then we select threshold value by using Equation (17).

$$
T^{*}=\sum_{z=0}^{255} z h(z)
$$

Based on the threshold value, the edge is evaluated from Equation (18).

$$
\operatorname{Edge}(x, y)= \begin{cases}\text { edge } & \text { if } M_{E}(x, y) \geq T^{*} \\ \text { not an edge } & \text { otherwise }\end{cases}
$$

\section{Experimental Results}

To demonstrate the effectiveness of the proposed method, we applied the proposed method to the image shown in Figure 6 and compared with the conventional methods(Sobel, Prewitt, and Canny). Moreover, in this letter, we have set $\alpha$ as $2 / 3$ which is used in [9].

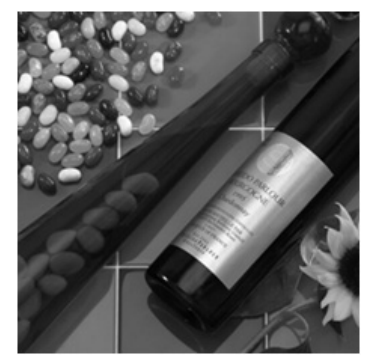

(a)image 1

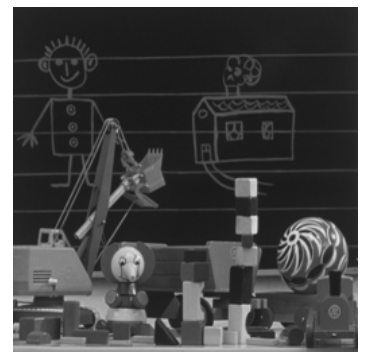

(c)image 3

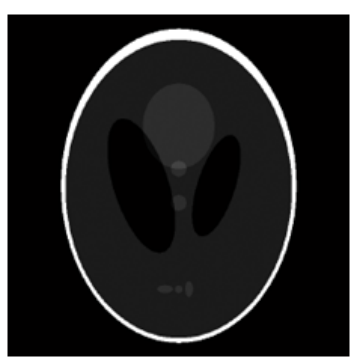

(b)image 2

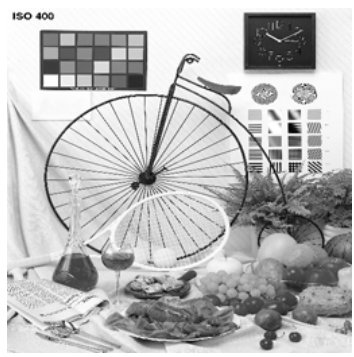

(d)image 4
Figure 6. Images used in experiment
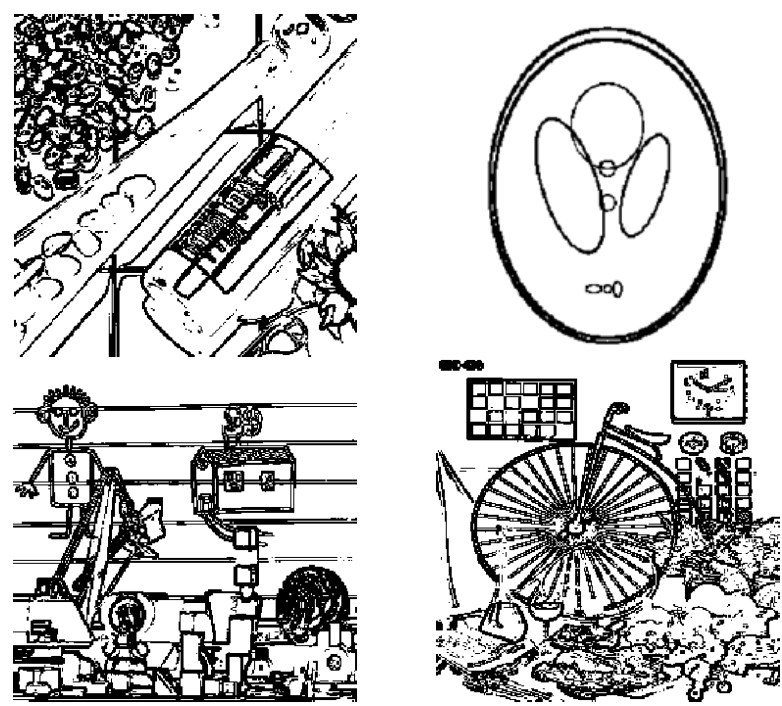

(a)Results of Sobel

where $h(z)$ is normalized histogram. 

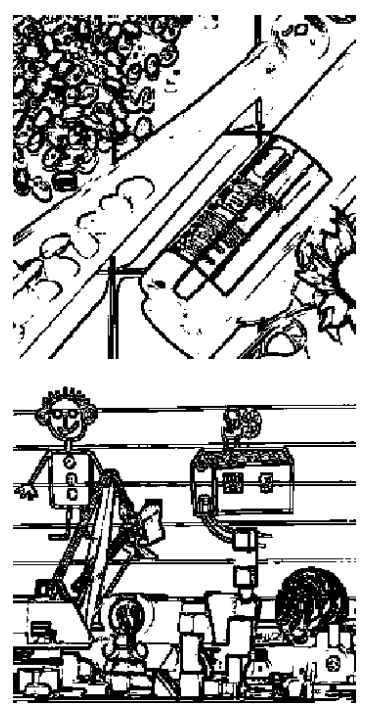

(b)Results of Prewitt
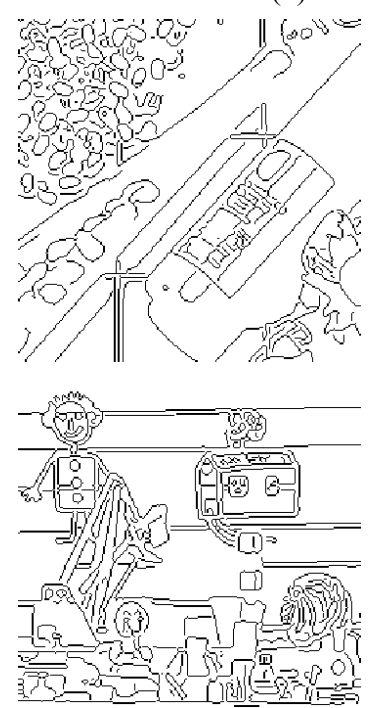

(c)Results of Canny
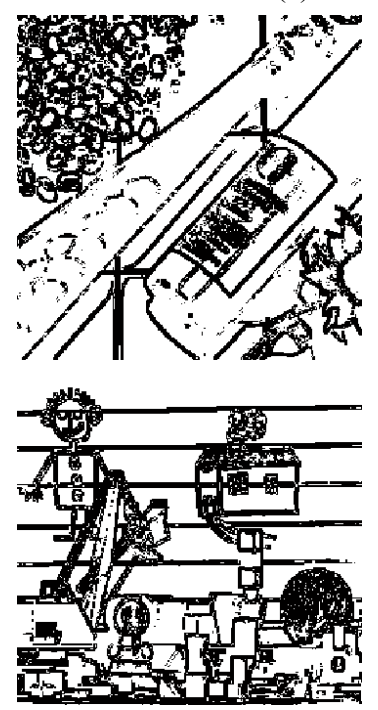

(d)Results of the proposed method
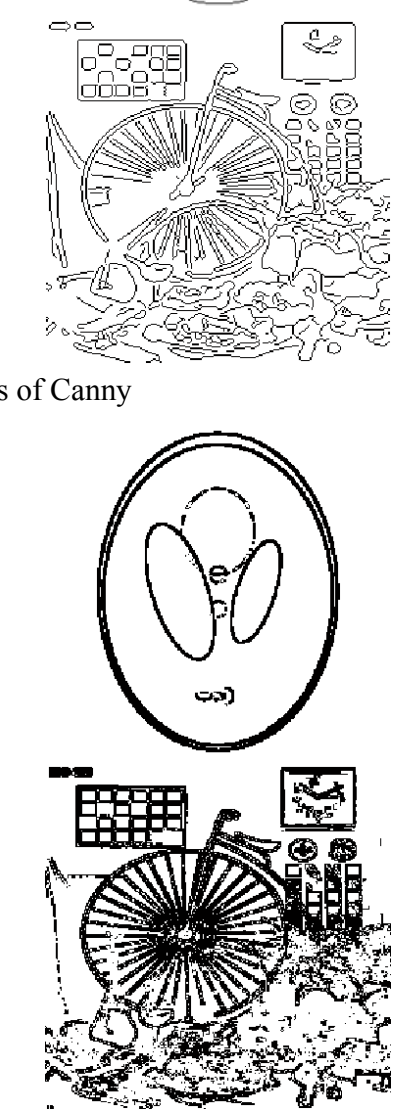

Figure 7. Experimental results
Figure 7 shows the results of edge detection of Figure 6 according to methods. From the results, Canny's method provides thinner edges than the other methods. However, when considering the absence of a thinning stage, the other methods are competitive with the Canny's method. Moreover, in some cases, Canny's method does not detect edges, especially, in wheel region of bike of Figure 6 (d).

For the proposed method, although it provides thicker edges than Sobel's and Prewitt's methods, the proposed method does not provide double edge lines for a single edge lines. Especially, for the results of Figure 6 (c), Sobel's and Prewitt's methods provides double edge lines for the background sigle horizontal edge lines of image while the propsoed method provides sigle lines.

From the overall results, although the proposed method does not provide the best results for all cases, it provides similar edge detection results to other edge detection methods. From the experimental results, we have shown that the possibility of edge detection based on the evidence theory.

\section{Conclusion}

In this paper, we have proposed an edge detection method based on evidence theory. Moreover, from the edge detection experiments, we have shown that edge detection method based on the Dempster-Shafer's evidence theory is effective to detect edges. As the further studies, researches for evidences to define edgeness and optimization method for $\alpha$ to improve the performance of the edge detection are required.

\section{References}

[1] R. C. Gonzalez, R. E. Woods, and S. L. Eddins, Digital Image Processing using MATLAB, Pearson, NJ, 2004.

[2] J. Canny, "A computational approach to edge detection", IEEE Trans. Pattern Analysis and Machine Intelligence, vol.8, no.6, pp.679-698, 1986.

[3] L. R. Liang and C. G. Looney, "Competitive fuzzy edge detection”, Applied Soft Computing, vol.3, no.2, pp.123-127, 2003.

[4] S. Zheng, J. Liu, and J. W. Tian, "A new efficient SVM based edge detection method", Pattern Recognition Lett., vol.25, no.10, pp.1143-1154, 2004.

[5] C. C. Kang and W. J. Wang, "A novel edge detection method based on the maximizing objective function", Pattern Recognition, vol.40, no.2, pp.609-618, 2007.

[6] G. Shafer, A Mathematical Theory of Evidence, Princeton University, 1976.

[7] Z. Chunjiang and D. Yong, "Color image edge detection using Dempster-Shafer theory”, Conf. 2009 Artificial Intelligence and Computational Intelligence, pp.476-479, 2009.

[8] Z. Chunjiang and D. Yong, "A modified Sobel edge detection 
using Dempster-Shafer theory", Conf. 2009 Image and Signal Processing, pp.1-4, 2009.

[9] T.-C. Lin, "Partition belief median filter based on DempsterShafer theory for image processing", Pattern Recognition, vol.41, no.1, pp.139-151, 2008.

[10] M. Rombaut and Y. M. Zhu, "Study of Dempster-Shafer theory for image segmentation applications", Image and Vision Computing, vol.20, no.1, pp.15-23, 2002.

[11] P. Vasseur, C. Pegard, E. Mouaddib, and L. Delahoche, "Perceptual organization approach based on Dempster-Shafer theory", Pattern Recognition, vol.32, no.8, pp.1449-1462, 1999.

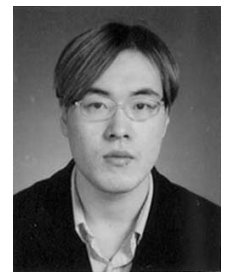

Suk Tae Seo received the B.S. and M.S. degrees in electrical engineering from Yeungnam University in 2004 and 2006 respectively. He obtained $\mathrm{PhD}$ in electrical engineering from Yeungnam University. His research interest include image processing, pattern recognition, image understanding and data mining.

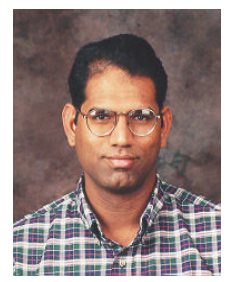

Krishnamoorthy Sivakumar received the B.Tech. degree in electrical engineering from the Indian Institute of Technology, Bombay, in 1991, and the M.S.E. degree in electrical and computer engineering, the M.S.E. degree in mathematical sciences, and the Ph.D. degree in electrical and computer engineering, all from the Johns Hopkins University, Baltimore, MD, in 1993, 1995 and 1997, respectively. He was a Visiting Assistant Professor with the Department of Electrical Engineering, Texas A\&M University, College Station, in 1997-1998. In 1998, he joined the School of Electrical Engineering and Computer Science at Washington State University, Pullman, where he is currently an Associate Professor. His research interests include statistical signal and image processing and data mining. He serves on the editorial board of the Journal of Mathematical Imaging and is a senior member of the IEEE.

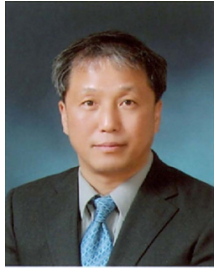

Soon Hak Kwon received the B.S. and the M.S. degrees in control engineering from Seoul National University in 1983 and 1985, respectively. He received the doctor of engineering degree from Tokyo Institute of Technology in 1995. He is now a full professor of Department of Electrical Engineering at Yeungnam University, where he has been with since 1996 and is now an adjunct faculty of EECS, WSU, USA. His research interests include Computational Vision, Information Mining, Image Processing, and Modeling of Intelligent Systems. 